\title{
Uso de regressão linear para estimar parâmetros físico-químicos relacionados à qualidade do jerked beef
}

Marcos Franke PINTO

Elisa Helena Giglio

PONSANO ${ }^{1}$

Ana Paula da Silva

ALMEIDA $^{1}$

Sílvia Helena Ventorulli

PERRI ${ }^{1}$

Massami SHIMOKOMAKI²

Correspondência para: MARCOSFRANKE PINTO

Departamento de Apoio, Produção e Ciência Animal.

R. Clóvis Pestana, 793

Caixa Postal 341

CEP: 16050-680 - Araçatuba-SP

mfpinto@fmva.unesp.br

Recebido para publicação: 30/08/2005 Aprovado para publicação: 13/02/2006

1 - Universidade Estadual Paulista, Araçatuba - SP

2 - Universidade Estadual de Londrina, Londrina - PR

\section{Resumo}

O jerked beefé oficialmente descrito como carne bovina salgada, curada e dessecada. Os parâmetros físico-químicos utilizados na determinação do seu padrão oficial de identidade e qualidade são a atividade de água e os teores de umidade, matéria mineral (ou cinzas) e nitrito residual. Neste trabalho, foi avaliada a evolução desses parâmetros durante o processamento do produto. Verificou-se haver uma correlação significativa entre eles, o que permitiu estabelecer equações estatísticas, que possibilitam estimar todos esses parâmetros através da aferição de apenas um deles.

\section{Introdução}

O jerked beef é um produto cárneo cuja tecnologia de processamento se assemelha à do charque tradicional, diferindo basicamente pela utilização de sais de cura nitrato e nitrito de sódio - na etapa de salga úmida e pela forma de adição da salmoura à carne, feita através de injetoras automáticas, enquanto, na fabricação do charque, esse processo é feito em tumblers ${ }^{1,2}$. Recentemente, o Ministério da Agricultura regulamentou o padrão de identidade e qualidade do jerked beef, definindo-o como um "produto cárneo industrializado obtido de carne bovina, adicionado de cloreto de sódio e sais de cura, submetido a um processo de maturação e dessecação". Assim como o charque, o jerked beef pode ser considerado um alimento de umidade intermediária. Segundo Leistner ${ }^{3,4}$, são assim classificados os alimentos que apresentam atividade de água entre 0,6 e 0,9, cuja estabilidade à temperatura ambiente se deve à ação de um ou mais fatores adicionais. No caso do jerked beef, esse fator adicional que, junto à baixa atividade de água, age como um obstáculo ao desenvolvimento microbiano, é representado pelo elevado teor de $\mathrm{NaCl}$. O Ministério da Agricultura ${ }^{5}$ estabeleceu, entre os critérios adotados para padronização do produto, as seguintes características físico-químicas: no máximo $55 \%$ de umidade, $18,3 \%$ de matéria mineral ou cinzas, $50 \mathrm{ppm}$ de nitrito residual e 0,78 de atividade de água. Para o monitoramento da qualidade do produto, buscando o atendimento de um mercado consumidor cada vez mais exigente e para a observância das especificações legais, as indústrias necessitam de métodos e ferramentas analíticas que proporcionem uma análise constante e de custo acessível.

\section{Materiais e Métodos}

Neste trabalho, foram coletadas amostras do músculo peitoral ascendente em 10 diferentes etapas da linha de processamento industrial do jerked beef (Industrias Allyson, Santana do Parnaíba/SP). De cada etapa, foram tomadas 3 amostras. Após retirada da gordura ostensiva, a porção muscular resultante foi triturada em aparelho multi-processador de alimentos e submetida à determinação, em triplicata, dos teores de umidade, cinzas, $\mathrm{NaCl}$ e $\mathrm{NaNO}_{2}{ }^{6}$. Também foi determinada a atividade de água das amostras, em equipamento Novasina modelo TH2/RD-33BDS.

Estatística descritiva foi realizada com 
os cálculos de médias e desvios padrões para cada variável estudada em cada etapa do processo de fabricação do jerked beef e coeficiente de correlação linear de Pearson entre as variáveis estudadas. Estatística inferencial foi realizada por meio de análise de regressão linear e análise de variância ${ }^{7}$.

\section{Resultados e Discussão}

A tabela 1 mostra o comportamento dos parâmetros físico-químicos estudados, durante o processamento de jerked beef. As médias observadas no produto final se enquadraram nos limites estipulados no padrão de identidade e qualidade do produto $^{5}$.

Os resultados demonstraram uma correlação significativa $(p<0,01)$ entre as variáveis estudadas (Tabela 2), o que permitiu formular equações de regressão linear para estimar todos os parâmetros legais através da determinação de apenas um deles (Tabelas 3 e 4$)$.

A tabela 2 mostra que os valores de umidade são correlacionados positivamente com os valores de atividade de água (Aw) e $\mathrm{NaNO}_{2}$, mas negativamente com os valores de cinzas e $\mathrm{NaCl}$. Esta significativa correlação $(\mathrm{p}<0,01)$ permitiu ajustar equações de regressão linear simples considerando umidade como variável independente de acordo com os seguintes modelos (Tabela 3):

1. Aw $=0,008$ umidade $+0,3496$

2. Cinzas $=-0,4043$ umidade $+38,725$

3. $\mathrm{NaCl}=-0,3686$ umidade $+35,149$

4. $\mathrm{NaNO}_{2}=1,6698$ umidade $-86,818$

De acordo com a ANOVA, a umidade pode explicar a variabilidade dos valores de $\mathrm{Aw}$, cinzas, $\mathrm{NaCl}$ e $\mathrm{NaNO}_{2}$ $(\mathrm{p}<0,01)$ com coeficientes de determinação $\mathrm{R}^{2}=88,4 \% ; 82,3 \% ; 76,7 \%$ e $43,3 \%$ respectivamente. A tabela 3 mostra os valores preditos e os respectivos intervalos de $95 \%$ de confiança para $50 \%$ e $55 \%$ de umidade.

Os valores de Aw são correlacionados positivamente com os valores de umidade e $\mathrm{NaNO}_{2}$, mas negativamente com os valores de cinzas e $\mathrm{NaCl}$ (Tabela 2). Esta significativa correlação $(\mathrm{p}<0,01)$ permitiu ajustar equações de regressão linear simples considerando Aw como variável independente de acordo com os seguintes modelos (Tabela 4):

1. Umidade $=110,93 \mathrm{Aw}-31,446$

2. Cinzas $=-47,994 \mathrm{Aw}+54,106$

3. $\mathrm{NaCl}=-44,195 \mathrm{Aw}+49,584$

4. $\mathrm{NaNO}_{2}=190,25 \mathrm{Aw}-143,58$

De acordo com a ANOVA, Aw pode explicar a variabilidade dos valores de umidade, cinzas, $\mathrm{NaCl}$ e $\mathrm{NaNO}_{2}(\mathrm{p}<0,01)$ com coeficientes de determinação $\mathrm{R}^{2}=$ $88,4 \% ; 83,2 \% ; 80,6 \%$ e $40,3 \%$ respectivamente. A tabela 4 mostra os valores preditos e os respectivos intervalos de 95\% de confiança para $0,75 \%$ e $0,78 \%$ de Aw.

Durante a elaboração de jerked beef, o nível máximo de nitrito foi atingido logo após a injeção da salmoura, e não ultrapassou $41 \mathrm{ppm}$. Durante o processamento, esse nível foi diminuindo, chegando a valores insignificantes no produto final (Tabela 1). Os baixos valores de nitrito residual observados no produto final fizeram com que, pela aplicação das equações de regressão, valores mais baixos de umidade e atividade de água correspondessem a valores de nitrito residual negativos (Tabelas 3 e 4). Na prática, observa-se que quando os parâmetros legais de atividade de água e umidade são atingidos, os valores de nitrito residual estão bastante reduzidos, devido às reações de degradação. Portanto, se respeitada a forma correta de processamento tecnológico, o nível de nitrito residual do produto não é um fator preocupante para sua qualidade. Quanto aos demais parâmetros legais, foi possível demonstrar que, entre os valores de 50 e $55 \%$ de umidade (Tabela 3), os valores preditos de cinzas e atividade de água, incluindo o intervalo de confiança, permanecem próximos dos limites estipulados pela legislação.

Observando a tabela 4, é possível obter conclusão semelhante, já que dentro do intervalo de 0,75 e 0,78 de atividade de água, considerando o intervalo de confiança, 
os valores determinados de cinzas e umidade permanecem dentro de uma faixa próxima dos padrões legais estabelecidos pelo Ministério da Agricultura ${ }^{5}$.

A aferição da umidade apresenta a vantagem de requerer equipamentos mais simples e de custo mais acessível. Já a avaliação da atividade de água permite obter resultados mais rapidamente, e o equipamento para essa aferição já é disponível em modelos portáteis, que fornecem leitura em 5 minutos ou menos. No entanto, seu custo é maior em relação aos equipamentos utilizados para a determinação da umidade. Desse modo, este trabalho propõe uma ferramenta matemática, que permite monitorar os parâmetros físico-químicos do produto durante o processamento, com maior facilidade, economia e rapidez.

\section{Conclusão}

Os parâmetros físico-químicos utilizados na definição legal do padrão de identidade e qualidade de jerked beef apresentam correlação significativa entre si, o que permite estimar vários parâmetros a partir da determinação do valor de apenas um deles, através de equações de regressão linear.

Tabela 1 - Evolução dos parâmetros físico-químicos durante o processo de fabricação do jerked beef (média \pm dp)

\begin{tabular}{|c|c|c|c|c|c|c|}
\hline $\begin{array}{c}\text { Etapa do } \\
\text { processa } \\
\text { mento }\end{array}$ & $\begin{array}{l}\text { Tempo de } \\
\text { proces- } \\
\text { samento } \\
\text { (dias) }\end{array}$ & $\begin{array}{c}\text { Umidade } \\
(\%)\end{array}$ & $\begin{array}{c}\text { Cinzas } \\
(\%)\end{array}$ & $\begin{array}{c}\mathrm{NaCl} \\
(\%)\end{array}$ & $\overline{A w}$ & $\begin{array}{c}\mathrm{NaNO}_{2} \\
\text { (ppm) }\end{array}$ \\
\hline $\begin{array}{l}\text { Matéria } \\
\text { prima }\end{array}$ & 0 & $76,7 \pm 0,7$ & $1,2 \pm 0,2$ & $0,2 \pm 0,1$ & $0,98 \pm 0,02$ & $3,1 \pm 2,8$ \\
\hline $\begin{array}{l}\text { Recém } \\
\text { injetada }\end{array}$ & 0 & $71,4 \pm 1,2$ & $8,6 \pm 0,6$ & $7,5 \pm 0,6$ & $0,95 \pm 0,03$ & $39,3 \pm 12,3$ \\
\hline $\begin{array}{l}\text { Salga } \\
\text { seca }\end{array}$ & 1 & $71,1 \pm 2,4$ & $9,9 \pm 1,1$ & $8,8 \pm 0,9$ & $0,92 \pm 0,02$ & $41,1 \pm 19,0$ \\
\hline Ressalga & 2 & $67,1 \pm 1,8$ & $10,9 \pm 2,1$ & $9,9 \pm 2,2$ & $0,90 \pm 0,05$ & $34,2 \pm 15,4$ \\
\hline $1^{\circ}$ tombo & 3 & $61,4 \pm 5,4$ & $14,2 \pm 1,5$ & $13,1 \pm 0,5$ & $0,85 \pm 0,03$ & $24,9 \pm 7,3$ \\
\hline $\begin{array}{l}\text { Final dos } \\
\text { tombos }\end{array}$ & 7 & $65,3 \pm 1,6$ & $13,4 \pm 1,1$ & $12,0 \pm 1,1$ & $0,87 \pm 0,03$ & $11,6 \pm 0,4$ \\
\hline $\begin{array}{l}\text { Após } \\
\text { lavagem }\end{array}$ & 8 & $66,8 \pm 1,6$ & $11,8 \pm 0,6$ & $10,9 \pm 0,3$ & $0,86 \pm 0,03$ & $13,4 \pm 5,2$ \\
\hline $\begin{array}{l}1^{\circ} \text { abafa- } \\
\text { mento }\end{array}$ & 10 & $56,7 \pm 1,6$ & $17,2 \pm 0,8$ & $15,5 \pm 1,0$ & $0,81 \pm 0,02$ & $4,0 \pm 0,6$ \\
\hline $2^{\circ} \mathrm{sol}$ & 12 & $62,9 \pm 2,3$ & $13,6 \pm 0,7$ & $12,3 \pm 0,8$ & $0,85 \pm 0,01$ & $5,6 \pm 0,7$ \\
\hline $\begin{array}{l}\text { Produto } \\
\text { final }\end{array}$ & 15 & $52,1 \pm 1,3$ & $18,1 \pm 0,9$ & $16,5 \pm 0,6$ & $0,75 \pm 0,01$ & $8,6 \pm 5,1$ \\
\hline \multicolumn{7}{|c|}{ ela $\angle$ - Lorrelaçao innear ae rearson entre as varravels esturaacas } \\
\hline Variáveis & \multicolumn{2}{|c|}{ Umidade } & Cinzas & $\mathbf{N a C l}$ & $\mathrm{NaNO}_{2}$ & Aw \\
\hline Umidade & \multicolumn{2}{|c|}{1,000} & & & & \\
\hline Cinzas & \multicolumn{2}{|c|}{$-0,907^{*}$} & 1,000 & & & \\
\hline $\mathrm{NaCl}$ & \multicolumn{2}{|c|}{$-0,876^{*}$} & $0,985^{*}$ & 1,000 & & \\
\hline $\mathrm{NaNO}_{2}$ & \multicolumn{2}{|c|}{$0,658^{*}$} & $-0,665^{*}$ & $-0,665^{*}$ & 1,000 & \\
\hline Aw & \multicolumn{2}{|c|}{$0,940^{*}$} & $-0,912^{*}$ & $-0,898^{*}$ & $0,635^{*}$ & 1,000 \\
\hline
\end{tabular}

${ }^{*} \mathrm{p}<0,01$ 
A aplicação dessas equações demonstra que os limites estabelecidos pela legislação são adequados e se ajustam ao comportamento dos parâmetros físico-químicos envolvidos.

Tabela 3 - Valores preditos, intervalo de confiança e equações de regressão das variáveis físico-químicas, de acordo com valores pré-determinados de umidade

\begin{tabular}{clcccc}
\hline $\begin{array}{c}\text { Valor de } \\
\text { Umidade } \\
(\%)\end{array}$ & Variável & $\begin{array}{c}\text { Valor } \\
\text { Predito }\end{array}$ & $\begin{array}{c}\text { Intervalo de } \\
\text { Confiança }\end{array}$ & Equação de Regressão \\
\hline \multirow{2}{*}{50} & $\mathrm{Aw}$ & 0,75 & 0,70 & 0,79 & $\mathrm{Aw}=0,008$ umidade $+0,3496$ \\
& $\mathrm{Cinzas}$ & 18,5 & 15,7 & 21,3 & $\mathrm{Cinzas}=-0,4043$ umidade $+38,725$ \\
& $\mathrm{NaCl}$ & 16,7 & 13,7 & 19,8 & $\mathrm{NaCl}=-0,3686$ umidade $+35,149$ \\
& $\mathrm{NaNO}$ & $-3,3$ & $-31,8$ & 25,2 & $\mathrm{NaNO}_{2}=1,6698$ umidade $-86,818$ \\
& $\mathrm{Aw}$ & 0,79 & 0,75 & 0,83 & $\mathrm{Aw}=0,008$ umidade $+0,3496$ \\
55 & $\mathrm{Cinzas}$ & 16,5 & 13,8 & 19,2 & $\mathrm{Cinzas}=-0,4043$ umidade $+38,725$ \\
& $\mathrm{NaCL}^{2}$ & 14,9 & 11,9 & 17,8 & $\mathrm{NaCl}=-0,3686$ umidade $+35,149$ \\
& $\mathrm{NaNO}_{2}$ & 5,0 & $-22,4$ & 32,5 & $\mathrm{NaNO}_{2}=1,6698$ umidade $-86,818$ \\
\hline
\end{tabular}

Tabela 4-Valores preditos, intervalos de confiança e equações de regressão das variáveis físico-químicas, de acordo com valores pré-determinados de Aw

\begin{tabular}{|c|c|c|c|c|c|}
\hline $\begin{array}{l}\text { Valor de } \\
\text { Aw (\%) }\end{array}$ & Variável & $\begin{array}{c}\text { Valor } \\
\text { Predito }\end{array}$ & \multicolumn{2}{|c|}{$\begin{array}{c}\text { Intervalo de } \\
\text { Confiança }\end{array}$} & Equação de Regressão \\
\hline \multirow{4}{*}{0,75} & Umidade & 51,8 & 46,7 & 56,8 & Umidade $=110,93 \mathrm{Aw}-31,446$ \\
\hline & Cinzas & 18,1 & 15,4 & 20,8 & Cinzas $=-47,994 \mathrm{Aw}+54,106$ \\
\hline & $\mathrm{NaCl}$ & 16,4 & 13,7 & 19,2 & $\mathrm{NaCl}=-44,195 \mathrm{Aw}+49,584$ \\
\hline & $\mathrm{NaNO}_{2}$ & $-0,90$ & $-29,9$ & 28,1 & $\mathrm{NaNO}_{2}=190,25 \mathrm{Aw}-143,58$ \\
\hline \multirow{4}{*}{0,78} & Umidade & 55,1 & 50,2 & 60,0 & Umidade $=110,93 \mathrm{Aw}-31,446$ \\
\hline & Cinzas & 16,7 & 14,0 & 19,3 & Cinzas $=-47,994 \mathrm{Aw}+54,106$ \\
\hline & $\mathrm{NaCl}$ & 15,1 & 12,4 & 17,8 & $\mathrm{NaCl}=-44,195 \mathrm{Aw}+49,584$ \\
\hline & $\mathrm{NaNO}_{2}$ & 4,8 & $-23,4$ & 33,0 & $\mathrm{NaNO}_{2}=190,25 \mathrm{Aw}-143,58$ \\
\hline
\end{tabular}

Use of linear regression to estimate physicochemical parameters associated to jerked beef quality

\section{Abstract}

Jerked beef is officially defined as salted, cured and dried beef. Water activity (Aw), moisture, ash and residual nitrite are the physicochemical parameters that define this product identity and quality standards. In this work, the behavior of these parameters during jerked beef processing was evaluated and a significant correlation among them was revealed. These results allowed the establishment of statistical equations that enable the estimation of all the physicochemical parameters from the results obtained in the measurement of just one of them.
Key words: Jerked beef. Linear regression. Quality control. Physicochemical parameters. 


\section{Referências}

1 SHIMOKOMAKI,M.; FRANCO, B. D. G.M.; BISCONTINI, T. M. B.; PINTO, M. F.; TERRA, N. N.;ZORN, T. M. T. Charqui meats are Hurdle Technology meat products. Food Rev. Int., New York, v. 14, n. 4, p. 339-349, 1998.

2 SHIMOKOMAKI,M.; FRANCO, B.D.G.M.; CARVALHOJr., B. C. Charque e produtos afins:tecnologia e conservação - uma revisão. Bol. Soc. Bras. Ciênc. Tecnol. Aliment., Campinas, v. 21, n. 1, p. 25-35, 1987

3 LEISTNER, L. Hurdle technology applied to meat products of the shelf stable products and intermediate moisture foods types. In: MULTON, J. L. Ed. Properties of water in foods. Dordrecht: Martinus Niyhoff, 1985. p. 309-329.

4 LEISTNER, L. Shelf stable products and intermediate moisture foods based on meats. In: ROCKLAND, L.B. and BEUCHAT, L. R. (Ed.). Water activity: theory and applications to food. N.York: Mercel Dekker, 1987. p. 295-327.

5 BRASIL. Portaria n. 3, de 17 de janeiro de 2000. Diário Oficial da União, Brasilia, 2000. Seção 1,p. 16-27.

6 (AOAC) - ASSOCIATION OF OFFICIAL ANALYTICAL CHEMISTS. Official methods of analysis. 13. ed. Washington: Association of Official Analytical Chemists, 1980.

7 ZAR, J. H. Biostatistical analysis. 4. ed. New Jersey: Prentice-Hall, 1998. 930 p. 